\title{
Investigations on domain boundaries in ordered GaInP using stereo transmission electron microscopy
}

\author{
G. Hahn, C. Geng†, P. Ernst, H. Schweizer, F. Scholz \\ 4. Phys. Institut, Universität Stuttgart, 70550 Stuttgart, Germany \\ F. PhillipP \\ Max-Planck-Institut für Metallforschung, Institut für Physik, Heisenbergstrasse 1, 70569 Stuttgart, \\ Germany
}

\begin{abstract}
$\mathrm{CuPt}_{B}$-type ordered GaInP, lattice matched to GaAs, has been grown by metal organic vapor phase epitaxy. Ordered domains and their boundaries are investigated by dark-field transmission electron microscopy. By means of stereo images, we are able to show that domain boundaries are microscopically thin. The fact that they generally appear as broad dark lines in dark-field images is due to the projection onto the image plane and does not mean that there is a disordered alloy surrounding ordered domains. This is in agreement with photoluminescence excitation spectra of ordered samples which do not show any sign of a disordered phase.
\end{abstract}

Key words: GaInP, ordering, order-domains, stereo-TEM.

\section{Introduction}

$\mathrm{CuPt}_{B}$-type ordering in GaInP has attracted a lot of interest in basic research, as well as for device applications. Although most optoelectronic devices are fabricated from rather disordered material [1, 2], ordered GaInP still has the potential of achieving superior laser diodes [3, 4]. An ideal ordered GaInP crystal would consist of a single sequence of Ga-rich and In-rich monolayers oriented along a [111 $]_{B}$ direction, characterized by a certain order parameter $\eta$ [5]. Previous investigations on the microstructure of experimentally obtained ordered layers, however, show that ordering occurs in so-called ordered domains [6-9]. These can be defined

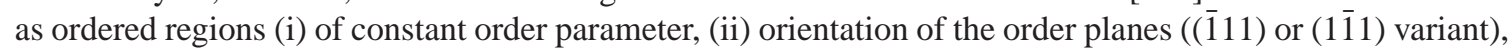
and (iii) undisturbed phase of the ordering superlattice. In order to obtain a better understanding of the actual physical properties of the ordered alloy, it is essential to understand the domain structure of ordered GaInP samples.

Domains may extend from about 5 to $500 \mathrm{~nm}$ and more in lateral width [4, 8, 9]. In dark-field transmission electron micrographs (DF-TEM), domain boundaries are represented by dark lines, which sometimes appear sharp, but are more usually diffuse and up to $50 \mathrm{~nm}$ thick. Up to now, two interpretations for these dark lines have been discussed:

$\dagger$ E-mail: christian.geng@temic.de 


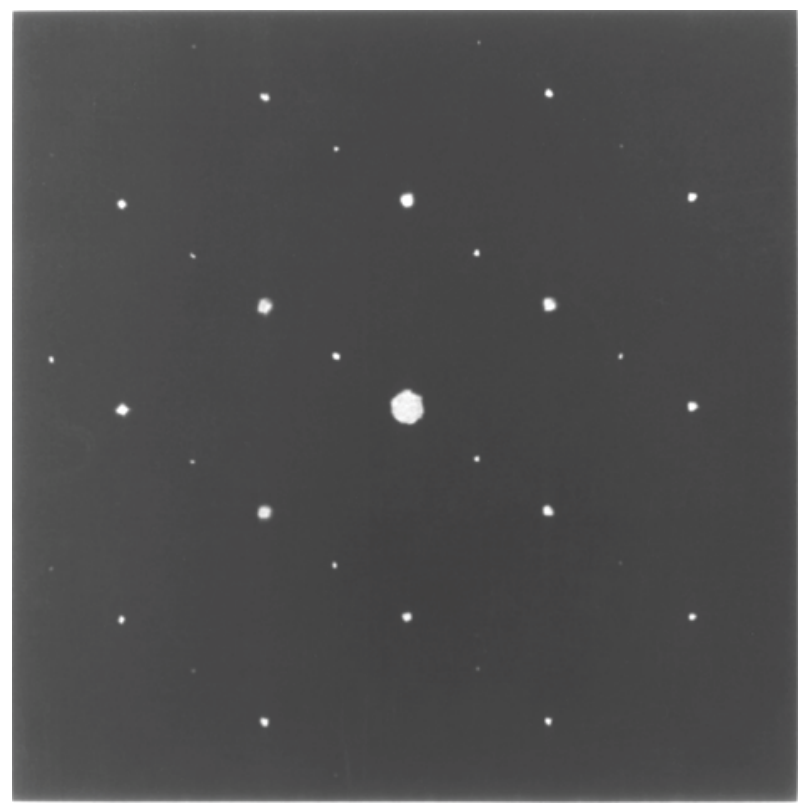

Fig. 1. Transmission electron diffraction pattern of the ordered GaInP sample in [110] cross section. The substrate was misoriented $6^{\circ}$ from (001) towards the ( $\left.\overline{1} 11\right)$ plane. As a consequence of the substrate tilt, only the (i11) order variant is present, showing itself in the intense superlattice reflections.

(i) They could represent disordered material. It has been proposed that the ordered crystal is like an 'emulsion' of ordered domains embedded in a matrix of disordered material [6, 10-12].

(ii) The ordering superlattice is shifted by half a superlattice period along a so-called anti-phase boundary (APB). The lines which appear in DF-TEM micrographs may then be atomically thin and the diffuse appearance is due to domain overlap [7, 8, 13, 14].

In this paper, we present data which allow one to discern between these two interpretations. By stereo TEM investigations, as well as optical spectroscopy, we show that microscopically thin APBs are usually projected onto the image plane.

We have grown GaInP, lattice matched to a (001) GaAs substrate, misoriented $6^{\circ}$ towards the (111) plane. The tilt to this group $\mathrm{V}$ face ( $B$-plane) is known to enhance ordering $[7,15]$ and to lead to a single ordering variant [6, 9] and, therefore, to a suppression of twin boundaries. Metal organic vapor phase epitaxy (MOVPE) at $100 \mathrm{hPa}$ in a horizontal reactor was used to grow the GaInP. At the applied growth temperature of $720^{\circ} \mathrm{C}$, this leads to an order parameter $\eta$ of 0.44 [16]. The sample was prepared for TEM as described in [9]. Figure 1 shows a diffraction pattern of this sample in [110] cross section. It is clearly observable in the intense superlattice reflections that, due to the substrate misorientation towards (111), only the ( $\overline{1} 11)$ variant occurs

Using the $\frac{1}{2}(1 \overline{1} 3)$ superlattice spot, dark-field images, as shown in Fig. 2, have been made. The ordered domains appear in bright contrast, whereas the domain boundaries remain dark. As expected for these growth parameters, the domains in this sample are about $200 \mathrm{~nm}$ wide, with a vertical extension of the layer thickness.

We now investigate the nature of the domain boundaries: The information of the micrograph originates from the sample volume, but only its two-dimensional projection is seen. Figures 2A and B show the same section of the sample. However, in between taking these pictures, the sample was tilted $15^{\circ}$ around the [1 $\left.\overline{1} 3\right]$ axis. In a stereo viewer, one may reconstruct a three-dimensional picture since the tilt angle is in harmony with the eye's parallax at the chosen magnification of $3 \times 10^{4}$. A detailed description of this method is given in $[17,18]$. The reader may try to experience the stereo effect by looking at Figs $2 \mathrm{~A}$ and B with the left and 


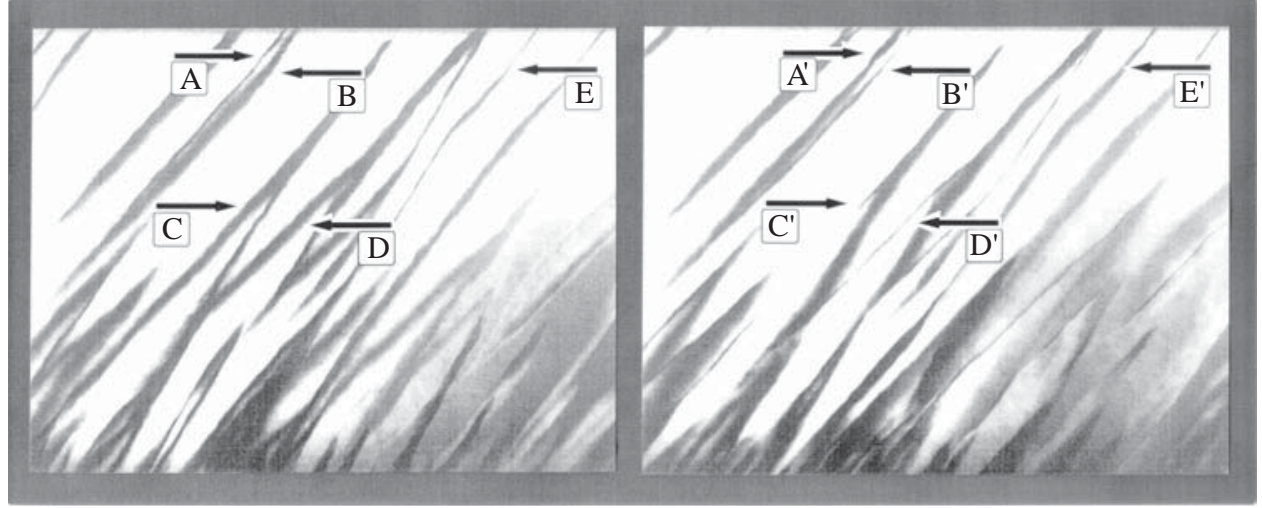

Fig. 2. Dark-field transmission electron micrograph using the $\frac{1}{2}(1 \overline{1} 3)$ superlattice spot. The ordered domains appear in bright contrast, whereas the domain boundaries remain dark. A and B have been taken at angles of electron beam incidence, differing $15^{\circ}$ around the [113] axis. This angle is supposed to be in harmony with the human eye's parallax, corresponding to the applied magnification of $3 \times 10^{4}$.

right eye respectively. One perceives an additional dimension (depth), allowing one to investigate the spatial arrangement of the domain boundaries in the epilayer. Even by looking separately at the pictures, we can describe the three-dimensional effect: the domain boundaries marked A and E come out very thin in Fig. 2A, running almost perpendicular to the picture plane. In Fig. 2B, these boundaries (marked as $\mathrm{A}^{\prime}$ and $\mathrm{E}^{\prime}$ ) appear thicker, due to the projection on the new plane of view. In reverse, $\mathrm{B}^{\prime}, \mathrm{C}^{\prime}$, and $\mathrm{D}^{\prime}$ appear thin in Fig. 2B, but in Fig. 2A, they give a thick dark contrast. So in $\mathrm{A}, \mathrm{B}^{\prime}, \mathrm{C}^{\prime}, \mathrm{D}^{\prime}$ ', and $\mathrm{E}$, we see domain boundaries 'accidentally' lying rather perpendicular to the picture plane, while in $\mathrm{A}^{\prime}, \mathrm{B}, \mathrm{C}, \mathrm{D}$, and $\mathrm{E}^{\prime}$ we are looking at them slightly from the side.

In the stereo view, the course of the domains is attended by the appearance of the wedge-like shape of the sample as prepared for TEM, which means that the sample is thin on the top of the picture, and becomes thicker towards the bottom. Therefore the plane-like domain boundaries that are not perfectly perpendicular to the plane of view appear thicker on the bottom of the picture than in the thin top of the sample prepared.

The stereo impression shows that domain boundaries have a well defined individual course in the epilayer. We conclude that domain boundaries have a rather infinitesimal volume. They almost always appear wider in the projection than they really are. Domain boundaries that are inclined to the plane of view appear dark due to the destructive interference between the diffracted beams from adjacent domains. This also confirms the anti-phase relationship across domain boundaries [7]. Although due to the limited resolution of this method (about $1 \mathrm{~nm}$ - due to the small apperture used), the atomic sharpness of the interfaces may not be proved, we suspect them to be even atomically sharp boundaries between domains of differently ordered phases.

We have to emphasize that in our sample only one variant is present. In some publications, reporting on double-variant samples [7, 14], a dark-field image may only be made by a superspot of one variant at a time. Therefore the regions where the other variant occurs will also remain dark. This will also lead to large dark regions in the image, which do not represent disordered bulk material either.

The fact that there is no 'disordered matrix' present in ordered GaInP samples can also be shown by optical spectroscopy, since the bandgap of the disordered material is higher than that of ordered material. Unfortunately, photoluminescence (PL) will not allow the detection of a disordered phase in the material, since photocreated carriers relax into regions of lower bandgap, i.e. of higher degree of ordering, instead of recombining within the disordered regions. By means of absorption spectroscopy or photoluminescence excitation spectroscopy (PLE), however, it would be possible to detect the disordered phase due to an increased absorption at the bandgap of disordered GaInP. The dark area covers about 10\% of the picture (see Fig. 2). 


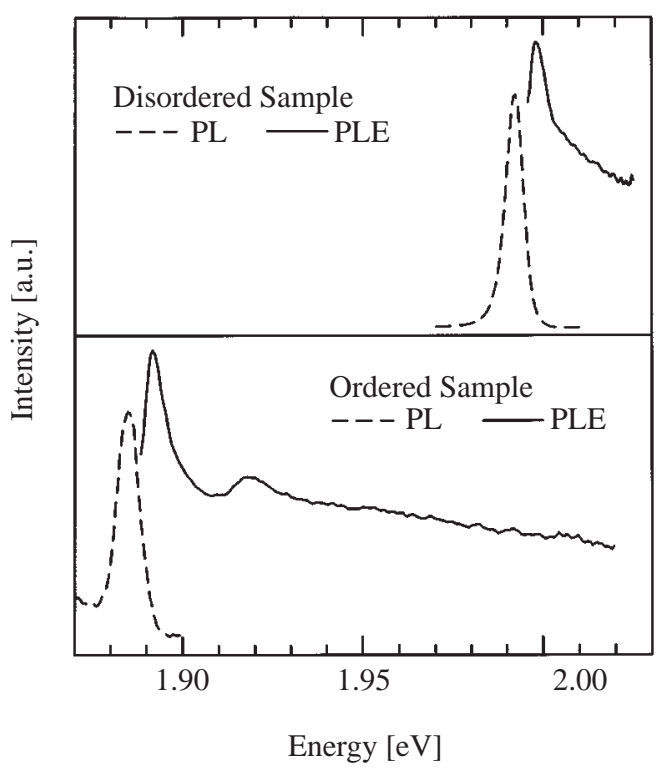

Fig. 3. PL (dashed) and PLE (solid) spectra from the ordered sample which was used for the TEM investigations and from a disordered reference sample. Spectra are taken at $T=2 \mathrm{~K}$.

If this was disordered material, its absorption should be observable, exceeding the absorption background of the ordered phase. In Fig. 3, we compare low-temperature PL and PLE spectra of the ordered sample which was used for the TEM micrographs and a completely disordered sample. Both PLE spectra show clear excitonic resonances close to the bandgap (at 1.892 and $1.998 \mathrm{eV}$ for the ordered and the disordered samples, respectively). Due to the valence band splitting, the ordered sample shows an additional resonance originating from excitons formed with the light-hole-like valence band (at $1.981 \mathrm{eV}$ ). The feature of interest, however, is that there is no increase in absorption nor any band-edge resonance present in the PLE of the ordered sample at photon energies corresponding to the bandgap of a hypothetical disordered phase. This is in agreement with our PLE spectra from several other samples with a large variety of growth conditions and degrees of order [9, 19].

In conclusion, we have shown that single-variant ordered GaInP samples do not contain any disordered phase. Instead, it can be concluded from stereo TEM images that the ordered phase fills the entire volume of the sample. Broad dark lines, which generally appear in dark field TEM images are projections of antiphase boundaries onto the image plane. They appear microscopically thin if observed edge-on in a suitable projection.

Acknowledgements—We would like to thank E. Kohler for technical assistance and M. H. Pilkuhn for stimulating discussions.

\section{References}

[1] I. Yoshida, T. Katsuyama, J. Hashimoto, Y. Taniguchi, and H. Hayashi, Electron. Lett. 28, 628 (1992).

[2] A. Valster, C. T. H. F. Lindenbaum, N. M. Finke, A. L. G. Severens, M. J. B. Boermans, D. W. W. Vandenhoudt, and C. W. T. Bulle-Liewma, J. Cryst. Growth 107, 403 (1991).

[3] A. Moritz and A. Hangleiter, Appl. Phys. Lett. 66, 3340 (1995). 
[4] C. Geng, A. Moritz, S. Heppel, A. Mühe, J. Kuhn, P. Ernst, H. Schweizer, F. Phillipp, A. Hangleiter, and F. Scholz, J. Cryst. Growth 107, 418 (1997).

[5] The degree of crystal ordering in GaInP is characterized by an order parameter $\eta$, denoting the distribution of gallium and indium on the $(111)_{B}$ sublattices according to: $\left(\mathrm{Ga}_{0.5(1+\eta)} \mathrm{In}_{0.5(1-\eta)} \mathrm{P} / \mathrm{Ga}_{0.5(1-\eta)} \operatorname{In}_{0.5(1+\eta)} \mathrm{P}\right)$.

[6] P. Bellon, J. P. Chevalier, E. Augarde, J. P. André, and G. P. Martin, J. Appl. Phys. 66, 2388 (1989).

[7] C. S. Baxter, W. M. Stobbs, and J. H. Wilkie, J. Cryst. Growth 112, 373 (1991).

[8] L. C. Su, I. H. Ho, and G. B. Stringfellow, J. Appl. Phys. 75, 5135 (1994).

[9] P. Ernst, C. Geng, G. Hahn, F. Scholz, H. Schweizer, F. Phillipp, and A. Mascarenhas, J. Appl. Phys. 79, 2633 (1996).

[10] G. J. Bauhuis, F. A. J. M. Driessen, and L. J. Giling, Phys. Rev. B48, 17239 (1993).

[11] F. A. J. M. Driessen, G. J. Bauhuijs, S. M. Olsthoorn, and L. J. Giling, Phys. Rev. B48, 7889 (1993).

[12] J. E. Fouquet, V. M. Robbins, S. J. Rosner, and O. Blum, Appl. Phys. Lett. 57, 1566 (1990).

[13] M. Ishimaru, S. Matsumura, N. Kuwano, and K. Oki, Phys. Rev. B51, 9707 (1995).

[14] L. C. Su, I. H. Ho, and G. B. Stringfellow, J. Appl. Phys. 76, 3520 (1994).

[15] S. R. Kurtz, J. M. Olson, D. J. Arent, M. H. Bode, and K. A. Bertness, J. Appl Phys. 75, 5110 (1994).

[16] P. Ernst, C. Geng, F. Scholz, H. Schweizer, Y. Zhang, and A. Mascarenhas, Appl. Phys. Lett. 67, 2347 (1995).

[17] J.F. Nankivell, Optik 20, 171 (1963).

[18] B. Hudson and M.J. Makin, J. Phys. E Scientific Instruments 3, 311 (1970).

[19] P. Ernst, C. Geng, F. Scholz, H. Schweizer, and A. Mascarenhas, Phys. Stat. Sol. (b) 193, 213 (1996). 